ISSN (e)-2347-176x ISSN (p) 2455-0450

crossrefDOI: https://dx.doi.org/10.18535/jmscr/v7i1.93

\title{
Assessment of imaging finding in Spinal tuberculosis
}

\author{
Authors \\ Dr Dipali Kadam, Dr Saurabh Patil ${ }^{2}$, Dr Avinash Dhok ${ }^{3}$, Dr Meenal Jain ${ }^{4}$ \\ ${ }^{1}$ Professor, Department of Radiology, NKPSIMS Nagpur \\ ${ }^{2,4}$ Junior Resident, Department of Radiology, NKPSIMS Nagpur \\ ${ }^{3}$ Professor \& HOD, Department of Radiology, NKPSIMS Nagpur \\ *Corresponding Author \\ Dr Saurabh Patil \\ Add: D-140, Saket, New Delhi.110017. India \\ Mobile No: 9730380000, Email: dr.slpatil24@gmail.com
}

\begin{abstract}
Introduction: Involvement of spine in tuberculosis is most common skeletal manifestation of extra-pulmonary tuberculosis. It is a destructive process involving abscess, collapse of vertebra, end plate irregularities, cord abnormalities and intervertebral disc deformity. X-ray always remains initial imaging modality of choice followed by CT and MRI. Most commonly followed path of assessment includes X-ray and MRI, as bony abnormalities seen best on X-ray and soft tissue abnormalities on MRI. Present study was performed to assess imaging findings in spinal tuberculosis using X-ray and MRI.

Method and Materials: The study was conducted on 50 patients clinically suspected of spinal tuberculosis referred to radiology department for further workup. After taking an informed and written consent, X-ray was taken first, followed by MRI.

Results: Back pain is most common symptom of Spinal TB. Kyphosis, scoliosis, collapse of vertebra, cord edema, involvement of disc, abscess, canal stenosis, reduced bone density and thecal sac indentation were the findings seen in spinal tuberculosis patients. MRI was noted to be a better modality for the evaluation of the canal abnormalities and extent of abscess.

Conclusion: X-ray remains initial imaging modality due to easily available cheap modality and excellent demonstration of bony abnormalities. However, MRI remains best available modality due to its excellent soft tissue demonstration and marrow lesions.
\end{abstract}

Keywords: Tuberculosis, Pott's spine, Extra-pulmonary, MRI, Spinal deformity.

\section{Introduction}

Spinal tuberculosis is a frequently encountered extra-pulmonary form of the disease. ${ }^{1}$ Mycobacterium tuberculosis is the main causative organism of tuberculosis. Early diagnosis and prompt treatment is necessary to prevent permanent neurological disability and to minimize spinal deformity. ${ }^{1}$ Approximately $2 \%$ of patients having tuberculosis have involvement of the skeletal system, while spinal tuberculosis being the most common in nearly $50 \%$ of all cases. ${ }^{2,3}$ Most commonly Spinal TB spread by haematogenous spread from pulmonary tuberculosis. The thoracic and lumbar spine with thoracolumbar junction are the most involved sites of spinal tuberculosis. The 
classic destruction of the disk space and the adjacent vertebral bodies, destruction of other spinal elements, severe and progressive kyphosis subsequently became known as Pott's disease, ${ }^{4}$ which currently describes tuberculous infection of the spine and the term 'Pott's paraplegia' describes paraplegia resulting from tuberculosis of the spine. ${ }^{5}$ The prognosis for spinal tuberculosis is improved by early diagnosis and rapid intervention. ${ }^{6} \mathrm{~A}$ high degree of clinical suspicion is required if patients present with chronic back pain, even in the absence of neurological symptoms and signs. ${ }^{7-8} \mathrm{MRI}$ is more sensitive and specific than X-ray and CT in establishing manifestations of tuberculosis, which helps in early diagnosis and better prognosis..$^{9-11}$

\section{Materials and Methods}

The present study was undertaken to assess imaging findings in spinal tuberculosis. The study was conducted on50 patients referred to radio-diagnosis department from November 2015 to September 2017 with clinically suspected spinal tuberculosis on the advice of referring doctor.

\section{Study design}

Cross sectional study over a period of 2 years.

\section{Inclusion criteria}

Patients who were referred with clinical history of spinal tuberculosis.

\section{Exclusion criteria}

1) Patients with a cardiac pacemaker

2) MRI non compatible stents and implants

3) Claustrophobic patients

\section{Methodology}

Those patients who fulfil the inclusion criteria and willing to participate in the study were selected on the basis of purposive sampling. A detailed history was taken with emphasis on duration of complaints. Informed and written consent of all cases was taken explaining the procedure.

\section{Imaging method}

- X-Ray: Both AP \& lateral X-rays were taken with patient erect position.

- MRI: HDXT 1.5 Tesla 16 channel- MRI machine
- Protocol:

$\begin{aligned} \text { i. } & \text { LOCALIZER_3PLANE } \\ \text { ii. } & \text { T2_TSE_SAG } \\ \text { iii. } & \text { T1_TSE_SAG } \\ \text { iv. } & \text { TSE_STIR_SAG } \\ \text { v. } & \text { Ax T1 } \\ \text { vi. } & \text { Ax T2* MERGE } \\ \text { vii. } & \text { TSE_STIR_COR }\end{aligned}$

Special sequences such as myelogram, gadoliniumenhanced T1-weighted images were also done whenever needed. All images were reviewed at an electronic PACS workstation by radiologists, and a consensus diagnosis was given by two radiologists in controversial cases.

\section{Statistical analysis}

The data on demography, x-ray, MRI and final diagnosis were obtained and analysed. Frequency distribution and percentage were obtained for age, gender, X-ray and MRI findings. The sensitivity, positive prediction value and accuracy were obtained for X-ray and MRI. Results were graphically represented in MS Excel 2010 where deemed necessary. All the analysis was performed using SPSS ver. 20.0 (IBM Corp.) software.

\section{Results}

Out of the 50 patients of our study, the age of the patients varied from second decade to sixth decade. Majority of the patients (22\%) were in the age group of 31-40 years followed by $20 \%$ in the age group of 41-50 years and in the age group of 21-30 years. $66 \%$ of the patients in our study were males, while female patients constituted $34 \%$ of the study population. The mean duration of symptoms was $16.45 \pm 12.3$ months. Most commonly, patients were presented with backache seen in $42(84 \%)$ patients, followed by fever noted in 37 (74\%) patients. Other symptoms were weakness, weight loss, bowel and bladder complaints. Thoracic spine is most commonly involved (72\%) site in our study, followed by thoraco-lumbar junction and lumbar spine. Cervical spine is least involved. Noncontiguous multiple level vertebral involvement also seen in 5 cases. Kyphosis, scoliosis, collapse of 
vertebra, cord edema, involvement of disc, abscess, canal stenosis, reduced bone density and thecal sac indentation were the findings seen in spinal tuberculosis patients. We compared X-ray and MRI findings as shown in following table.

Table 1: Comparison of findings in spinal tuberculosis

\begin{tabular}{|c|c|c|c|}
\hline Findings & & X-ray & MRI \\
\hline \multirow{3}{*}{ Kyphosis } & Sensitivity (\%) & 100 & 100 \\
\hline & PPV (\%) & 100 & 100 \\
\hline & Accuracy $(\%)$ & 100 & 100 \\
\hline \multirow{3}{*}{ Scoliosis } & Sensitivity (\%) & 100 & 100 \\
\hline & PPV (\%) & 100 & 100 \\
\hline & Accuracy $(\%)$ & 100 & 100 \\
\hline \multirow{3}{*}{$\begin{array}{l}\text { Collapse of } \\
\text { vertebra }\end{array}$} & Sensitivity (\%) & 100 & 100 \\
\hline & PPV (\%) & 100 & 100 \\
\hline & Accuracy $(\%)$ & 100 & 100 \\
\hline \multirow{3}{*}{ Cord edema } & Sensitivity (\%) & - & 100 \\
\hline & PPV (\%) & - & 100 \\
\hline & Accuracy $(\%)$ & - & 100 \\
\hline \multirow{3}{*}{$\begin{array}{l}\text { Disc } \\
\text { involvement }\end{array}$} & Sensitivity (\%) & 36 & 100 \\
\hline & PPV (\%) & 100 & 100 \\
\hline & Accuracy $(\%)$ & 36 & 100 \\
\hline \multirow{3}{*}{ Abscess } & Sensitivity (\%) & 68 & 100 \\
\hline & PPV (\%) & 100 & 100 \\
\hline & Accuracy (\%) & 68 & 100 \\
\hline \multirow{3}{*}{ Canal stenosis } & Sensitivity (\%) & 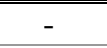 & 100 \\
\hline & PPV (\%) & - & 100 \\
\hline & Accuracy $(\%)$ & - & 100 \\
\hline \multirow{3}{*}{$\begin{array}{l}\text { Reduced bone } \\
\text { density }\end{array}$} & Sensitivity (\%) & 100 & - \\
\hline & PPV (\%) & 100 & - \\
\hline & Accuracy $(\%)$ & 100 & - \\
\hline \multirow{3}{*}{$\begin{array}{l}\text { Thecal sac } \\
\text { indentation }\end{array}$} & Sensitivity (\%) & - & 100 \\
\hline & PPV (\%) & - & 100 \\
\hline & Accuracy $(\%)$ & - & 100 \\
\hline
\end{tabular}

\section{Discussion}

Spinal involvement of tuberculosis is most destructive process affecting mostly children and young generation. Nearly half of the skeletal involvement of tuberculosis patients have spinal tuberculosis. $^{12}$ Etiological factors ofspinal tuberculosis are poverty, malnutrition, alcohol drinking, drug abuse, diabetes mellitus, immunosuppressive patients, and $\mathrm{HIV}{ }^{13}$ Haematogenous spread of infection into vascular cancellous bone of the vertebral bodiesis route of transfer $M$. tuberculosis into spine. Initial involvement of anterior inferior portion of the vertebral body followed by spreads into the central part of the body or disk. Characteristic features of spinal tuberculosis include kyphosis, scoliosis, destruction of the intervertebral disk space and the adjacent vertebral bodies, collapse of the vertebral body and elements, and gibbus formation. Thoracic spine followed by lumbar are most frequently involved sites. ${ }^{14}$

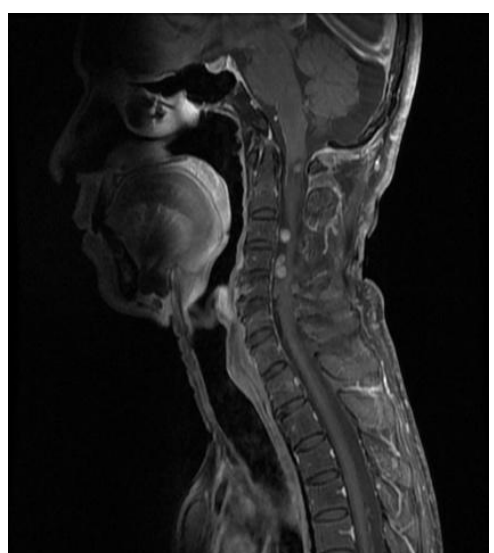

Fig 1: Multiple ring enhancing lesions in the intradural extramedullary compartment of cervical spine at the level of $\mathrm{C} 3$ and $\mathrm{C} 4$ vertebral bodies.

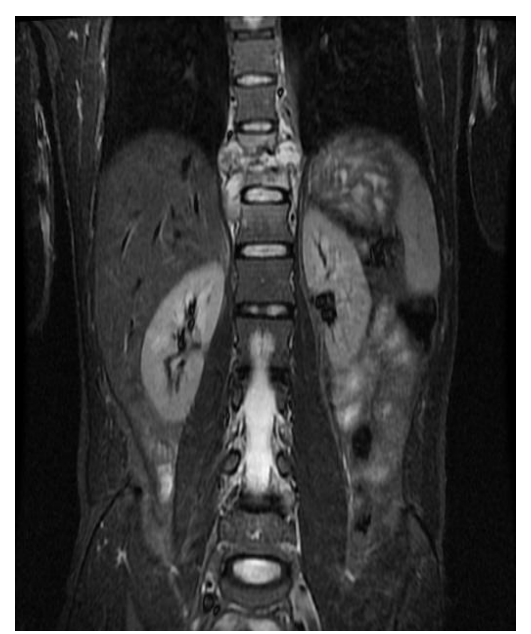

Fig 2: Subligamentus para-spinal collection in pre and para vertebral location at T10 and T11 vertebral levels.

In our study, majority of the patients $(22 \%)$ were in the age group of $31-40$ years followed by $20 \%$ in the age group of 41-50 years and in the age group of 21-30 years. No gender prediction seen. As par study done by Cormican L, Hammal R, Messenger J, Milburn HJ on spinal tuberculosis, classical constitutional features of tuberculosis were malaise, loss of weight and appetite, night sweats, evening rise in temperature, generalized body aches, and fatigue. Similar findings we observed in our study. 
Back pain is the most common complaint of spinal TB patient presented with. Bowel and bladder complains are due to neurological involvement of the disease. This may lead to complete paraplegia or tetraplegia. Fever, weakness, tenderness are other symptoms of spinal tuberculosis.

Initial evaluation of disease starts with X-ray. Bony abnormalities are best seen on X-ray and CT scan. $\mathrm{X}$-ray findings seen in tuberculosis patients in our study was Kyphosis, scoliosis, collapse of vertebra, involvement of disc, abscess andreduced bone density. However, cord is not seen on X-ray, so abnormal cord findings are missed on X-ray. Also exact extent of abscess, Para-spinal muscle involvement was not seen on X-ray. Similar findings were observed by Hodgson AR, Wong W, $\mathrm{Yau}^{16}$ in a study of spinal tuberculosis using X-ray as imaging modality.

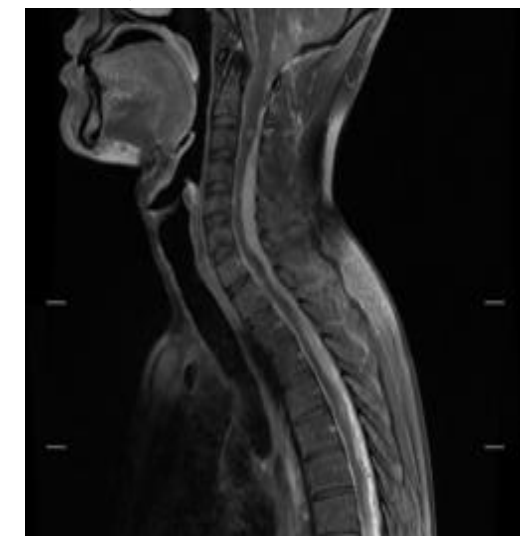

Fig 3: Enhancing exudates in the posterior subarachnoid space, in the region of cervical and upper thoracic spine

Additional to these X-ray findings cord edema, extent of abscess, intervertebral disc changes were excellently demonstrated on MRI. However, reduced bone densities were missed on MRI.End plate irregularity was also easily seen on MRI as compared to plain X-Rays. Wedging, compression fractures and vertebral body height reduction were equally imaged on X-ray and MRI. Abscess Formation near vertebral lesion is frequently characteristic feature of spinal tuberculosis. From very small sized to very large size abscess was seen in spinal TB. This abscess tracks down to the mediastinum and further extent into the trachea, oesophagus, or the pleural cavity. Retropharyngeal abscess may result in pressure effects such as dysphagia, respiratory distress, or hoarseness of voice. Thoracic abscess presents as a paravertebral swellings and seen as a posterior mediastinal mass. Another imaging finding, we saw on MRI was noncontinuous involvement of vertebral bodies. As seen in figure 5, on post contrast study of MRI, multiple enhancing lesions were noted on multiple vertebrae. It is an atypical form of spinal tuberculosis that affects two non-contiguous vertebrae without destruction of the adjacent vertebral bodies and intervertebral disks.

Our study concluded that, MRI delivers highest diagnostic accuracy in differentiating various disease processes. Its superior soft tissue differentiation and ability to visualize and detect lesions within the bone marrow, the spinal cord and the intervertebral disc makes MRI superior over other imaging modalities. Similar studies done by Ansari $\mathrm{S}$ et $\mathrm{al}^{17}$ also noted that MRI was better than $\mathrm{X}$-Rays to visualize spinal tuberculosis assessment.

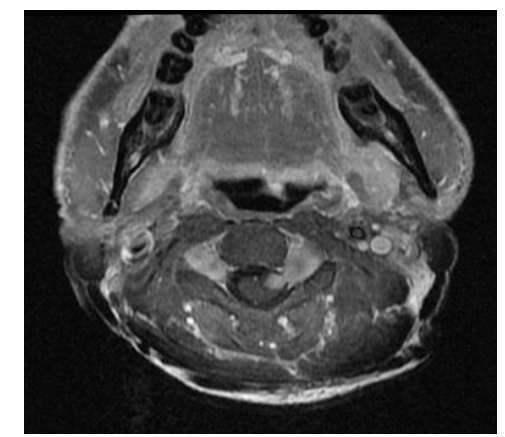

Fig 4: Ring enhancing lesions in the intra dural extramedullary compartment of cervical spine at the level of C3 vertebra.

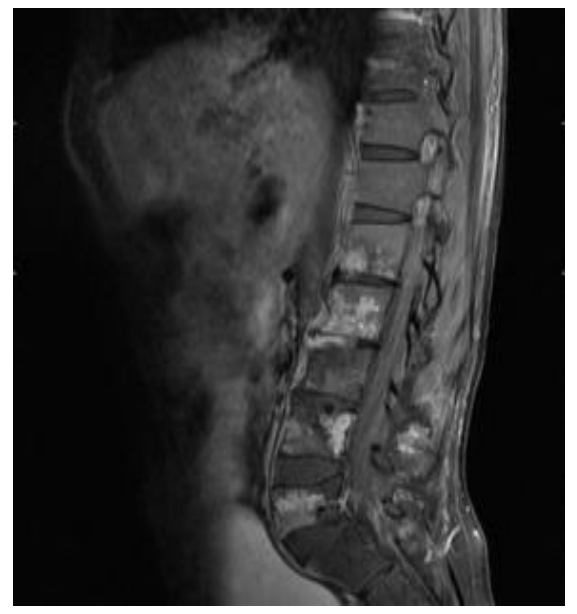

Fig 5: Multiple enhancing areas (abscesses) are seen at all the lumbar vertebral bodies. Distal spread of infection at L2-L3 disc level. 


\section{Conclusions}

The thoracic region of vertebral column is most frequently affected in spinal tuberculosis.X-ray is the initial imaging modality of choice as it is cheap, easily available and better for demonstration of bony abnormalities. However, magnetic resonance imaging is more sensitive imaging technique than $\mathrm{x}$ ray and more specific than computed tomography for the diagnosis of spinal tuberculosis. Magnetic resonance imaging frequently demonstrates involvement of the vertebral bodies on either side of the disk, disk destruction, cold abscess, vertebral collapse, and presence of vertebral column deformities. Hence it is absolutely necessary to evaluate every patient with clinical suspension of spinal tuberculosis with MRI.

\section{References}

1. World Health Organization. TB Facts 2011 [assessed on 2011 Jun 11].

2. Agrawal V, Patgaonkar PR, Nagariya SP. Tuberculosis of spine. J Craniovertebr Junction Spine. 2010;1:74-85.

3. Tuli SM. 3rd ed. New Delhi: Jaypee Brothers; 2004. Textbook- Tuberculosis of the skeletal system (Bones, Joints, Spine and Bursal sheaths)

4. World Health Organization. 2010/2011 tuberculosis global facts. Facts sheet no 104, 2010 Nov [accessed on 2011 Apr 16].

5. Taylor GM, Murphy E, Hopkins R, Rutland $\mathrm{P}$, Chistov Y. First report of Mycobacterium bovis DNA in human remains from the Iron Age. Microbiology 2007;153(4):1243-9 [PubMed]

6. Jain AK. Tuberculosis of the spine: a fresh look at an old disease. J Bone Joint Surg Br 2010;92(7):905-13 [PubMed]

7. Pawar UM, Kundnani V, Agashe V, Nene A, Nene A. Multidrug-resistant tuberculosis of the spine - is it the beginning of the end? A study of twenty-five culture proven multidrug-resistant tuberculosis spine patients. Spine (Phila $\mathrm{Pa}$ 1976) 2009;34(22):E806-10 [PubMed]
8. Talbot JC, Bismil Q, Saralaya D, Newton DA, Frizzel RM, Shaw DL. Musculoskeletal tuberculosis in Bradford - a 6-year review. Ann R Coll Surg Engl 2007;89(4):405-9 [PMC free article] [PubMed]

9. Omari B, Robertson JM, Nelson RJ, Chiu LC. Pott's disease. A resurgent challenge to the thoracic surgeon. Chest. 1989;95:145150 .

10. Shanley DJ. Tuberculosis of the spine: Imaging features. Am J Roentgenol 1995;164:659-64.

11. Griffith JF, Kumta SM, Leung PC, Cheng JC, Chow LT, Metreweli C. Imaging of musculoskeletal tuberculosis: a new look at an old disease. Clin Orthop Relat Res 2002;398:32-9.

12. Garg RK, Somvanshi DS. Spinal tuberculosis: a review. J Spinal Cord Med. 2011;34(5):440-54.

13. McLain RF, Isada C. Spinal tuberculosis deserves a place on the radar screen. Cleve Clin J Med2004;71(7):543-9

14. Moon MS. Tuberculosis of the spine. Controversies and a new challenge. Spine (Phila Pa 1976)1997;22(15):1791-7

15. Cormican L, Hammal R, Messenger J, Milburn HJ. Current difficulties in the diagnosis and management of spinal tuberculosis. Postgrad Med J 2006;82(963):46-51

16. Hodgson AR, Wong W, Yau A. X-ray appearance of tuberculosis of the spine. Springfield, IL: Charles C Thomas; 1969

17. Ansari S, Amanullah MF, Ahmad K, Rauniyar RK. Pott's Spine: Diagnostic Imaging Modalities and Technology Advancements. N Am J Med Sci. 2013; 5:404-411. 